\title{
The mediating role of KITLG DNA methylation in the association between childhood adversity and cortisol stress reactivity does not replicate in monocytes
}

\author{
Leonard Frach ${ }^{1}$, Sascha Tierling ${ }^{2}$, Marion Schwaiger ${ }^{1}$, Dirk Moser ${ }^{1}$, Markus Heinrichs ${ }^{3,4}$, Jan \\ G. Hengstler ${ }^{5}$, Jörn Walter ${ }^{2}$, Robert Kumsta ${ }^{1 *}$
}

${ }^{1}$ Department of Genetic Psychology, Faculty of Psychology, Ruhr University Bochum, Bochum, Germany

${ }^{2}$ Department of Genetics/ Epigenetics, Saarland University, Saarbrücken, Germany.

${ }^{3}$ Department of Psychology, Laboratory for Biological and Personality Psychology, University of Freiburg, Freiburg, Germany

${ }^{4}$ Freiburg Brain Imaging Center, University Medical Center, University of Freiburg, Freiburg, Germany ${ }^{5}$ Leibniz Research Centre for Working Environment and Human Factors at the Technical University of Dortmund (IfADo), Dortmund, Germany

Short title: DNA methylation and cortisol stress reactivity

Number of words:

Number of tables: 2

Number of figures: 4

\section{${ }^{*}$ Corresponding address:}

Prof Dr. Robert Kumsta

Department of Genetic Psychology

IB 5/117

Ruhr-University Bochum

Universitätsstraße 150

44780 Bochum, Germany

Phone: +49 2343222676

E-mail: robert.kumsta@rub.de 


\section{Abstract}

Adverse childhood experiences such as maltreatment or neglect are associated with mental health problems in adulthood. Changes in the regulation of the psychological and physiological stress reaction, mediated via epigenetic modifications, are discussed as potential mechanisms. This study aimed to replicate the role of DNA methylation of the KITLG gene in mediating the association between childhood adversity and stress-induced cortisol reactivity in a sample of adults reporting childhood adversity and a matched control group ( $N=60)$. DNA was extracted from purified $\mathrm{CD}_{14}{ }^{+}$monocytes and genome-wide DNA methylation was assessed with the 450k BeadChip for targeted replication and exploratory analyses. As previously reported, childhood adversity was associated with significantly lower cortisol reactivity to stress. We could neither replicate the association between KITLG DNA methylation and cortisol stress reactivity nor the association with childhood adversity. Moreover, DNA methylation of the target CpG (cg27512205) was not associated with KITLG mRNA expression in monocytes. Exploratory analyses of array-wide DNA methylation patterns showed no significant results for individual sites after correction for multiple testing neither in association with childhood trauma nor with adult cortisol stress reactivity. The analysis of differentially methylated regions (DMRs) revealed two significant regions which both mapped to non-coding genes in the association with cortisol stress reactivity.

The mediating role of DNA methylation of the KITLG locus in the association between childhood adversity and cortisol stress reactivity could not be replicated in monocytes. In addition to differences in investigated tissue, reasons for non-replication might include differences between samples in age, ethnicity, trauma severity, and cortisol reactivity.

Keywords: DNA Methylation, childhood adversity, EWAS, stress, TSST, replication 
PsyArXiv post-print doi: 10.31234/osf.io/6ed7n This is the accepted manuscript now published in Psychoneuroendocrinology https://doi.org/10.1016/j.psyneuen.2020.104653. This work is licensed under a CC-BY-NC-ND 4.0 International license.

\section{Introduction}

Severe psychosocial adversity in childhood such as exposure to physical or sexual abuse, neglect or institutional deprivation is associated with poor mental health and neurodevelopmental difficulties later in life [Gilbert et al., 2009; Sonuga-Barke et al., 2017]. This raises the question of how the long-lasting health consequences of early adverse environments are sustained. Models addressing the question of how the experience of early adversity becomes 'biologically embedded' assume stable alterations in structure and function of different regulatory systems, including those involved in executive functions of the prefrontal cortex, emotion processing, affiliative processes, the immune system, and stress regulation [Hertzman, 2012]. Among these target systems, the hypothalamic-pituitaryadrenal (HPA) axis, the organism's major neuroendocrine stress system, has been studied most extensively. A large body of research has shown dysregulations of the HPA axis in children and adults who were exposed to deprivation or abuse [Koss and Gunnar, 2018; Kumsta et al., 2017; Lupien et al., 2009], and in turn, alteration in HPA axis control and function have been associated with increased risk for a range of mental health problems [Chrousos, 2009], suggesting a mediating role of this stress system in the link between adverse childhood experience and disease risk in adulthood [Heim et al., 2008]. Reports investigating changes in HPA axis function following early adversity have assessed both, stress reactivity and basal - or unstimulated - activity. Following psychosocial stress exposure, reduced HPA axis reactivity has been observed in the majority of studies [Carpenter et al., 2007; Elzinga et al., 2008; Lovallo et al., 2011; MacMillan et al., 2009; Power et al., 2012; Schwaiger et al., 2016], although increased HPA axis responses have also been reported [Heim et al., 2000].

In the search for the mechanisms underlying long-lasting alterations of HPA axis function, and more generally, increased disorder risk following the experience of adversity, epigenetic modifications such as DNA methylation have emerged as potential mediators. Research using rodent models has shown that the extent of maternal care determined DNA methylation patterns in the regulatory regions of several genes involved in the control of the HPA axis, including Crf [Chen et al., 2012], Avp [Murgatroyd et al., 2009], and Nr3c1 [Weaver et al., 2004]. Low maternal care and the respective epigenetic changes were associated with HPA axis hypersensitivity and impaired negative HPA axis feedback sensitivity.

Using post-mortem brain tissue, non-experimental studies in humans provide evidence for similar epigenetic alterations of the NR3C1 promoter region in individuals exposed to adversity early in life [Labonte et al., 2012; McGowan et al., 2009]. Furthermore, a growing body of research has shown associations between early adversity and altered DNA methylation in non-neuronal cells of NR3C1 [Argentieri et al., 2017; reviewed by Turecki and Meaney, 2016] and other genes involved in HPA axis regulation [Klengel et al., 2013; Non et al., 2016]. 
Given that the effects of the early environment on epigenetic modifications are unlikely to be limited to genes involved in direct HPA axis control, a recent investigation performed an epigenome-wide screen to identify differentially methylated CpG sites associated with stress reactivity. Houtepen et al. [2016] found an association between whole blood DNA methylation of one $\mathrm{CpG}$ at the Kit ligand gene (KITLG) locus which was associated with the cortisol stress response to the Trier Social Stress Test (TSST), and could replicate the finding in two independent samples (one using whole blood, one buccal cells). Importantly, KITLG DNA methylation mediated to a considerable extent the link between childhood trauma and the cortisol stress response, although this was only observed in the discovery sample. KITLG codes for a ligand of tyrosine-kinase receptor encoded by the KIT locus. It is involved in fundamental processes of cellular development such as hematopoiesis [Su et al., 2013], neurogenesis and neuroprotection [Zhao et al., 2007]. There is some evidence for a possible role of KITLG in HPA axis regulation. One study found an association between early life stress and hippocampal Kitlg expression in mice [Suri et al., 2014]. In human cord blood, KITLG was found to regulate the expression of NR3C1 gene following induced erythropoiesis [Varricchio et al., 2012].

One aim of the study was to replicate the association between KITLG DNA methylation and cortisol reactivity, as well as its mediating role between childhood trauma and the cortisol stress response, taking advantage of available data from a study that used the same instrument to assess childhood adversity, the same psychosocial stress protocol, and the same array to quantify DNA methylation levels. In contrast to the previous report, we used a homogenous cell population, namely $\mathrm{CD} 14^{+}$monocytes. We chose to analyze monocytes as previous studies have shown that among the heterogeneous leukocyte population, monocytes were the most sensitive subtype for social conditions and traumatic experiences, at least in terms of transcriptional alterations following adversity [Cole et al., 2012; Cole et al., 2011; O'Donovan et al., 2011; Powell et al., 2013]. Another aim of the study was to explore associations between DNA methylation, childhood trauma and cortisol stress reactivity, respectively, using an epigenome-wide association analysis.

\section{Materials and Methods}

\section{Sample Characteristics}

The sample consisted of 60 healthy adults aged between 39 and 60 years who were recruited via articles in local newspapers and community-posted flyers. The German 28-item version of the Childhood Trauma Questionnaire [CTQ, Rodewald, 2005] was used to assess five categories of childhood adversities (sexual, physical and emotional abuse, as well as physical and emotional neglect). In order to classify subjects as positive for a history of childhood 
adversity, CTQ cut-off scores for moderate to severe exposure to traumatic experiences were used. Experience of adversity was validated in a structured interview with the Early Trauma Inventory [ETI, Heim, 2000]. Participants who met the criteria for mental disorders at the time of assessment or during the preceding 12 months (screened for with Structured Clinical Interview for DSM Disorders [SKID I, Wittchen et al., 1996] were excluded from study participation. The control group consisted of 30 participants who scored below cutoff on all CTQ subscales, and who were matched for gender, age, and current SES as well as childhood SES. For both groups, the use of psychoactive medication or hormone intake (e.g. oral contraceptives) led to study exclusion. Participants were paid $100 €$ for participation. The participants gave written informed consent to the study procedures, and the study was approved by the ethics committee of the Albert-Ludwigs University Freiburg (183/11). The study was part of a larger project investigating the long-term consequences of childhood adversity, which included the assessment of hormonal and genomic responses to stress, and the investigation of emotion recognition abilities [Schwaiger et al., 2016; Schwaiger et al., 2018].

\section{Stress Response Measures}

Psychosocial stress was induced with the TSST, a standardized 15-min stress protocol, which consists of a mock job interview and an unanticipated mental arithmetic task [see Kirschbaum, 1993]. All experimental sessions started at 2 p.m. Blood samples for the analyses of ACTH and cortisol were drawn via an indwelling catheter at 45 and 2 min prior, and 1, 10, 20, 30, 45, and $90 \mathrm{~min}$ post exposure to the TSST. Total cortisol and ACTH concentrations were measured with an enzyme-linked immunosorbent assay (IBL, Germany) at the University of Trier. Interassay and intrassay coefficients of variation were both under $6.9 \%$. As previously reported, General Linear Models were computed to assess the repeated measures effect time, the between-subjects effect group as well as the interaction time $\mathrm{x}$ group for endocrine and subjective responses to the TSST exposure. Greenhouse-Geisser corrections were applied where appropriate, and only adjusted results are reported. In order to compare our results with those reported by Houtepen et al. [2016], and in order to derive a continuous composite cortisol stress measure for DNA methylation analyses, we used the area under the cortisol response curve with respect to the increase [AUCi; Pruessner et al., 2003]. The AUCi was calculated using six time points (45 minutes before the TSST and one minute, 10, 20, 30 and 90 minutes after the TSST). One participant was excluded due to missing cortisol values. We also calculated a baseline-to-peak measure by subtracting the value 45 minutes before the TSST from the individual peak level in cortisol following the TSST as a further indicator of the cortisol stress response. 
PsyArXiv post-print doi: 10.31234/osf.io/6ed7n This is the accepted manuscript now published in Psychoneuroendocrinology

\section{DNA Methylation and mRNA Expression}

DNA was extracted from $\mathrm{CD}_{14}{ }^{+}$monocytes isolated via immunomagnetic cell separation (MACS; Miltenyi Biotec, Germany). Purity of the isolated monocyte population was checked with fluorescence-activated cell sorting analyses and showed high purity values (mean = 92.92\%, SE = 0.59 [Schwaiger et al., 2016]; see also Supplemental Figure 1 for estimation of cell composition). Nonetheless, we statistically corrected for the cell-type composition of the samples in the linear models. Genomic DNA was treated with sodium bisulfite using the EZ96 DNA Methylation Kit (Zymo Research) following the manufacturers' standard protocol. DNA methylation was quantified using the Illumina Infinium HumanMethylation450 BeadChip using an Illumina HiScan System at the University of Saarbrücken. The samples were randomized with respect to group status to avoid batch effects. Illumina Genome Studio software was used to extract the raw signal intensities of each probe. RNA was extracted from CD14 ${ }^{+}$monocytes isolated from EDTA blood samples collected at $45 \mathrm{~min}$ before, as well as 45 and $180 \mathrm{~min}$ after the TSST. Genome-wide transcriptional profiling was performed on the Agilent Whole Human Genome Oligo Microarrays $8 \times 60 \mathrm{~K}$ V2. All samples were randomized within and between arrays to avoid potential batch effects. The assays were performed at the Molecular Service Center (Miltenyi Biotech) following the manufacturer's standard protocol. Quantile-normalized gene expression values were log2-transformed for further analyses. See Schwaiger et al. (2016) for details. For targeted analysis of KITLG mRNA expression, we used the only available KITLG probe on the array (Agilent probe ID: A_24_P133253, RefSeq accession number: NM_000899).

\section{Statistical Analyses}

All analyses were performed using $R$ version 3.6.1 [R Core Team, 2014], and preprocessing and statistical analyses were aligned to those used by Houtepen et al. (2016).

For data import, preprocessing and analyses of DNAm we used the minfi [Aryee et al., 2014], sva [Leek et al., 2018], wateRmelon [Pidsley et al., 2013], DMRScan [Page et al., 2018], bumphunter [Jaffe et al., 2012] and limma packages [Ritchie et al., 2015] from the Bioconductor platform [Huber et al., 2015] and followed the workflow of Maksimovic et al. [2016]. Age and sex were included as covariates in all linear models.

\section{Preprocessing}

Quality control of array-wide DNA methylation with the minfi package indicated that none of the participants had more than $1 \%$ of failed probes or a mean detection $p$-value $>0.001 .4,850$ probes with a detection $p$-value $>0.001$ in $1 \%$ of samples and 279 probes with a bead count $<3$ in $5 \%$ of samples were removed using the wateRmelon package. Furthermore, probes located on sex chromosomes, probes with SNPs within 10 base pairs of the primer with a 
minor allele frequency $>5 \%$ and cross-reactive probes were removed, resulting in a final set of 408,145 CpGs.

DNA methylation was examined using the m-values, which meet the assumptions of homoscedasticity of residuals [Du et al., 2010]. The beta-values were used for graphical presentation of DNA methylation. BMIQ normalization was performed using the wateRmelon package as in the original study. Comparison of the non-normalized and the normalized data highlighted one participant as an outlier, which was excluded from all analyses.

MDS plots of principal components were inspected visually for identification of batch effects (array and position) using the minfi package. We corrected for the previously identified batch effects using the ComBat procedure as implemented in the sva package.

\section{Replication Analysis}

For replication analysis, 19 CpGs annotated to the KITLG locus, including target cg27512205, were retrieved. The association between DNA methylation of cg27512205 and stress reactivity, and the association between childhood adversity and DNA methylation of the CpG of interest was tested with linear regression models including age, sex and cell composition as covariates. Subsequently, linear regression models were run for all $\mathrm{CpGs}$ annotated to KITLG. Mediation analysis was performed using the mediation package [Tingley et al., 2014], a Quasi-Bayesian approach with 10,000 simulations as well subsequent sensitivity analysis, highlighting potential non-considered factors which influence both the mediator (DNAm) and the outcome (stress reactivity). As the mediation package is designed for the analysis of categorical predictors, we used the group variable (childhood adversity vs controls) as predictor instead of creating arbitrary groups based on total CTQ score.

\section{Additional Analysis}

We further investigated association between DNAm levels of the KITLG locus and the mRNA expression levels at baseline. We analysed whether the groups differed in baseline as well as stress-induced KITLG mRNA expression by investigating a $2 \times 3$ ANOVA with the betweensubject factor group (EA vs. CG) and the within-subject factor time.

\section{Exploratory Analysis}

We investigated array-wide DNA methylation in association with childhood adversity and adult cortisol stress reactivity using the limma package as in the original study and using the minfi package for confirmation. Because of the potential mediating effects of DNAm on the association between childhood trauma and adult stress reactivity, we first looked at the association between childhood trauma (predictor) and DNAm (mediator), which differs from 
the approach of Houtepen et al. (2016), who first investigated the association between DNA methylation and cortisol stress reactivity. We additionally investigated differentially methylated regions (DMRs) using the bumphunter [Jaffe et al., 2012] and DMRScan [Page et al., 2018] packages.

\section{Results}

\section{Cortisol Stress Reactivity}

Mean CTQ score of the early adversity group was 67.1 compared to 35.5 in the control group $\left(t_{37.14}=-10.1, p<0.001\right)$. Frequencies of the experienced types of trauma in the early adversity group are shown in Supplemental Table 1). As reported previously [Schwaiger et al., 2016], the early adversity group showed significantly lower cortisol responses to the TSST (main effect group: $F_{1,56}=5.97, p=0.018, \eta^{2}=0.076$; interaction: $F_{6,336}=3.13, p=0.005, \eta^{2}=$ 0.013). There was a negative association between the total CTQ score and the cortisol AUCi $(\beta=-0.251, p=0.064)$, used as a composite stress measure to enable comparison with Houtepen et al. (2016). Furthermore, the TSST was associated with increased self-reported psychological stress, measured by tense arousal, self-directed emotions, and anxiety; all F > 2.86, all $p<0.03$, all $\eta 2>0.013$. However, there were no differences between the two groups, nor was there an interaction between group by time (all $F<1.8$, all $p>0.18$; all $F<0.42$, all $p$ $>0.79$, respectively).

\section{DNA Methylation}

The association between childhood adversity and DNA methylation of the target KITLG cg27512205 could not be replicated, using both the CTQ score as a continuous measure ( $\beta=$ $-0.032, p=0.817$; Figure $1 \mathrm{~A})$, or the dichotomous group variable $\left(\mathrm{t}_{56}=-0.082, p=0.935\right)$.

Furthermore, the association between cg27512205 DNA methylation and adult cortisol stress reactivity could not be replicated ( $\beta=-0.061, p=0.653$; Figure $1 B$ ). The baseline-to-peak of the cortisol reaction as additional indicator of the cortisol stress response did also not reveal an association with DNA methylation of KITLG $\operatorname{cg} 27512205$ ( $\beta=-0.062, p=0.653$ ).

Results of the mediation analysis (Figure 2) showed a significant direct effect between childhood adversity and adult stress reactivity $(p=0.009)$, but no mediating effect of cg27512205 DNA methylation ( $p=0.974 ; 0.05 \%$ mediation). We extended our analysis to all other CpGs annotated to the KITLG locus and found that none of the 19 remaining CpGs showed a significant association with total CTQ scores or with the cortisol AUCi (Figure 3) at gene-wide threshold $(p=0.00263)$. 
To test for potential functional effects of $\operatorname{cg} 27512205$ DNA methylation on gene expression, we tested the association between DNA methylation of the KITLG target CpG and mRNA expression at baseline. However, the majority of the sample (69\%) showed very low KITLG mRNA expression levels (-log2 value $<0.1$ ), thus the associations are reported only visually (Supplemental Figure 2). Furthermore, changes in KITLG gene expression were not associated with changes in cortisol stress reactivity in both groups (EA: $r=-0.11, p=0.56$; CG: $r=-0.26, p=0.18$; see Supplemental Figure 3).

\section{Exploratory Analysis}

Like Houtepen et al. (2016), we did not find any significant differentially methylated positions (DMPs) in association with both CTQ and cortisol AUCi after False Discovery Rate (FDR) correction. Table 1 shows the ten strongest associations (based on p-value) between DMPs and CTQ scores $(A)$ and the ten strongest associations between DMPs and Cortisol AUCi (B). The investigation of differentially methylated regions revealed two significant DMRs, each comprised of two CpGs, in the association with adult cortisol stress reactivity (Figure 4), but not with childhood trauma. There were no significant DMRs when using the package bumphunter. Both DMRs are annotated to non-coding genes (LINC01725/ MIR548AP and LOC101929241) and no mRNA expression levels of corresponding transcripts were available. Moreover, we found no mediating role of these DMRs in the association between childhood adversity and stress reactivity, as the DNAm patterns were not related to childhood trauma.

\section{Discussion}

Epigenetic modifications have been discussed as a potential mechanism involved in the link between exposure to childhood adversity and altered stress reactivity in adulthood. Animal studies as well as human studies have provided first evidence for an association between altered DNA methylation of genes important for HPA axis regulation and cortisol stress reactivity. Using an unbiased genome-wide approach, DNA methylation at the KITLG locus in two tissues - whole blood and buccal cells - was recently found associated with the cortisol stress response [Houtepen et al., 2016]. Furthermore, it was shown that the link between childhood adversity and cortisol reactivity was mediated via KITLG DNA methylation. This was found in the discovery sample only, whereas the two replication samples could confirm the association between DNA methylation and the cortisol stress response, but not the association between childhood adversity and DNA methylation, nor the mediation effect.

The aim of the current study was to replicate these findings using a highly similar design, albeit assessing DNA methylation in a homogeneous cell population (isolated CD14+ monocytes), to control for the major confound of cellular heterogeneity [Jaffe and Irizarry, 2014]. The 
second aim was to perform an exploratory EWAS to uncover possible new associations between DNA methylation and childhood adversity and adult cortisol stress reactivity, respectively.

Whereas the present study provided additional support for the well-established association between childhood adversity and long-term alteration in HPA axis reactivity, we could not replicate the finding of KITLG locus DNA methylation as a mediator between childhood trauma and adult stress reactivity. Furthermore, there was no association between childhood adversity and KITLG DNA methylation, and no association between KITLG DNA methylation and cortisol stress reactivity. Several factors might explain the diverging results, as the present study differed from the original discovery and the two replication samples in several aspects, including age of participants, severity of trauma exposure, cortisol measures, and investigated tissue (Table 2). Thus, the present study cannot be regarded as an exact replication, but rather a conceptual one.

\section{Differences between the studies}

Participants in this study were recruited based on the experience of severe early adversity. This differs from the recruiting procedure of the discovery sample and replication sample 2 , where convenience samples were used. This explains the comparably much lower CTQ values reflecting much lower degree of childhood adversity in these cohorts compared to the present sample. The first replication sample included some individuals with exposure to childhood trauma, with similar mean CTQ scores and ranges compared to the present sample. With regard to cortisol AUCi values, the discrepancy between studies can be explained by different biomaterial for cortisol assessment, i.e. saliva in discovery and replication 2, and serum in replication sample 1 and the present study. The major difference between the studies is certainly the studied tissue. In our study, DNA methylation levels were assessed in in purified $\mathrm{CD} 14^{+}$monocytes, whereas the previous studies used more heterogenous tissues with cell type-specific DNA methylation levels [Reinius et al., 2012]. Houtepen et al. (2016) performed a statistical correction for cell type composition, however, it is still debated whether these methods can fully account for cellular heterogeneity [Jaffe and Irizarry, 2014; Marabita et al., 2013].

\section{Statistical Power}

Given the small sample size, low statistical power could also explain the missing associations between DNAm of the KITLG locus and CTQ as well as with cortisol AUCi. However, the 
reported effects from Houtepen et al. (2016) are strong (model fit: $R^{2}=0.34$ ) so that we had sufficient power to detect such effects $(1-\beta=0.97)$.

\section{Exploratory array-wide analyses}

Exploratory analysis showed no significant differentially methylated positions after FDR correction associated with childhood adversity or cortisol reactivity. As the power to identify small effects at single CpGs sites was small, we also conducted regional analyses, which utilize patterns of co-correlation between nearby CpG sites and require less power [Jaffe et al., 2012]. The two identified DMRs all mapped to long intergenic non-protein coding RNA, whose function remains obscure. One previous study reported hypermethylation of a DMR around LOC101929241 associated with increased prenatal phthalate exposure [Solomon et al., 2017].

\section{Conclusions}

In conclusion, the recently reported mediating role of DNA methylation in the association between childhood adversity and cortisol stress reactivity could not be confirmed in the present study. It is of note that Houtepen et al. (2016) observed mediation by KITLG DNA methylation in their discovery only, not in their replication samples, and did not find an association between KITLG DNA methylation and childhood adversity in two more recent large population-based cohorts [Houtepen et al., 2018].

Using whole blood comprising DNA from multiple cell types, each with a specific DNA methylation profile, leads to the challenge of controlling the influence of differential proportions of these cell types. On the other hand, when the primary tissue of interest is the brain, and easily accessible tissue such as blood or buccal cells are merely used as biomarkers, the question of which cells might be specifically affected is not of interest. The conclusion that can be drawn from this study is that monocytes do not appear to be good biomarkers for the effects of childhood adversity on KITLG DNA methylation differences. It cannot be ruled out that the effect reported by Houtepen et al. (2016) is real but occurs in another cell type than CD14 ${ }^{+}$ monocytes. It would have been desirable to examine more than one immune subpopulation which is a limitation of the present study - to identify the specific cell type, or alternatively a broad signature across the majority of immune cells, as target for the effects of early adversity.

The exploratory array-wide analyses provided little support for strong associations between childhood adversity and DNA methylation differences, but our sample was not powered to identify small effects. Future studies with larger sample sizes analyzing defined cell types as well as collaborative efforts to combine existing studies with available DNA methylation and 
PsyArXiv post-print doi: 10.31234/osf.io/6ed7n This is the accepted manuscript now published in Psychoneuroendocrinology https://doi.org/10.1016/j.psyneuen.2020.104653. This work is licensed under a CC-BY-NC-ND 4.0 International license.

cortisol stress reactivity data are warranted to conduct robustly powered EWAS. Furthermore, prospective-longitudinal studies, where DNA methylation is assessed proximal to the exposure of childhood adversity and not years to decades later, might increase chances of identifying a mediating role of epigenetic alterations in the long-term effects of early adversity.

\section{Funding and Disclosure}

This work was supported by a project grant by the German Research Foundation (DFG) to RK (KU 2479/3-1, KU 2479/3-2). The DFG has no role in study design, collection, analysis and interpretation of data, writing of the manuscript or in the decision to submit the paper for publication. All authors reported no biomedical financial interests or potential conflicts of interest.

\section{References}

Argentieri MA, Nagarajan S, Seddighzadeh B, et al.: Epigenetic Pathways in Human Disease: The Impact of DNA Methylation on Stress-Related Pathogenesis and Current Challenges in Biomarker Development. EBioMedicine 2017;18:327-350.

Aryee MJ, Jaffe AE, Corrada-Bravo $\mathrm{H}$, et al.: Minfi: a flexible and comprehensive Bioconductor package for the analysis of Infinium DNA methylation microarrays. Bioinformatics 2014;30:1363-1369.

Carpenter L, Carvalho J, Tyrka A, et al.: Decreased Adrenocorticotropic Hormone and Cortisol Responses to Stress in Healthy Adults Reporting Significant Childhood Maltreatment. Biological Psychiatry 2007;62:1080-1087.

Chen J, Evans AN, Liu Y, et al.: Maternal deprivation in rats is associated with corticotrophin-releasing hormone $(\mathrm{CRH})$ promoter hypomethylation and enhances $\mathrm{CRH}$ transcriptional responses to stress in adulthood. Journal of neuroendocrinology 2012;24:1055-1064.

Chrousos GP: Stress and disorders of the stress system. Nat Rev Endocrinol 2009;5:374381.

Cole SW, Conti G, Arevalo JM, et al.: Transcriptional modulation of the developing immune system by early life social adversity. Proc Natl Acad Sci U S A 2012;109:20578-20583.

Cole SW, Hawkley LC, Arevalo JM, et al.: Transcript origin analysis identifies antigenpresenting cells as primary targets of socially regulated gene expression in leukocytes. Proc Natl Acad Sci U S A 2011;108:3080-3085.

Du P, Zhang X, Huang CC, et al.: Comparison of Beta-value and M-value methods for quantifying methylation levels by microarray analysis. BMC Bioinformatics 2010;11:587.

Elzinga BM, Roelofs K, Tollenaar MS, et al.: Diminished cortisol responses to psychosocial stress associated with lifetime adverse events a study among healthy young subjects. Psychoneuroendocrinology 2008;33:227-237.

Gilbert R, Widom CS, Browne K, et al.: Burden and consequences of child maltreatment in high-income countries. Lancet 2009;373:68-81.

Heim C: Deutsche Version des Early Trauma Inventory: Inventar zur Erfassung früher traumatischer Lebensereignisse (IFTL). Unveröffentliches Manuskript, Department of Psychiatry and Behavioral Sciences, Emory University School of Medicine, Atlanta, GA, USA 2000.

Heim C, Newport DJ, Heit S, et al.: Pituitary-adrenal and autonomic responses to stress in women after sexual and physical abuse in childhood. Jama 2000;284:592-597. 
PsyArXiv post-print doi: 10.31234/osf.io/6ed7n This is the accepted manuscript now published in Psychoneuroendocrinology https://doi.org/10.1016/j.psyneuen.2020.104653. This work is licensed under a CC-BY-NC-ND 4.0 International license.

Heim C, Newport DJ, Mletzko T, et al.: The link between childhood trauma and depression: insights from HPA axis studies in humans. Psychoneuroendocrinology 2008;33:693710.

Hertzman C: Putting the concept of biological embedding in historical perspective. Proc Natl Acad Sci U S A 2012;109 Suppl 2:17160-17167.

Houtepen LC, Hardy R, Maddock J, et al.: Childhood adversity and DNA methylation in two population-based cohorts. Transl Psychiatry 2018;8:266.

Houtepen LC, Vinkers CH, Carrillo-Roa T, et al.: Genome-wide DNA methylation levels and altered cortisol stress reactivity following childhood trauma in humans. Nat Commun 2016:7:10967.

Huber W, Carey VJ, Gentleman R, et al.: Orchestrating high-throughput genomic analysis with Bioconductor. Nat Methods 2015;12:115-121.

Jaffe AE, Irizarry RA: Accounting for cellular heterogeneity is critical in epigenome-wide association studies. Genome Biol 2014;15.

Jaffe $A E$, Murakami $P$, Lee $H$, et al.: Bump hunting to identify differentially methylated regions in epigenetic epidemiology studies. Int J Epidemiol 2012;41:200-209.

Kirschbaum C, Pirke, K. M. Hellhammer, D. H.: The 'Trier Social Stress Test '- a tool for investigating psychobiology stress responses in a laboratory setting. Neuropsychobiology 1993;28:76-81.

Klengel T, Mehta D, Anacker C, et al.: Allele-specific FKBP5 DNA demethylation mediates gene-childhood trauma interactions. Nat Neurosci 2013;16:33-41.

Koss KJ, Gunnar MR: Annual Research Review: Early adversity, the hypothalamic-pituitaryadrenocortical axis, and child psychopathology. J Child Psychol Psychiatry 2018;59:327-346.

Kumsta R, Schlotz W, Golm D, et al.: HPA axis dysregulation in adult adoptees twenty years after severe institutional deprivation in childhood. Psychoneuroendocrino 2017;86:196202.

Labonte B, Yerko V, Gross J, et al.: Differential glucocorticoid receptor exon 1(B), 1(C), and $1(\mathrm{H})$ expression and methylation in suicide completers with a history of childhood abuse. Biological psychiatry 2012;72:41-48.

Leek JT, Johnson WE, Parkerl HS, et al.: va: Surrogate Variable Analysis. R package version 3.28.0. 2018.

Lovallo WR, Farag NH, Sorocco KH, et al.: Lifetime Adversity Leads to Blunted Stress Axis Reactivity: Studies from the Oklahoma Family Health Patterns Project. Biological Psychiatry 2011;71:344-349.

Lupien SJ, McEwen BS, Gunnar MR, et al.: Effects of stress throughout the lifespan on the brain, behaviour and cognition. Nat Rev Neurosci 2009;10:434-445.

MacMillan HL, Georgiades K, Duku EK, et al.: Cortisol response to stress in female youths exposed to childhood maltreatment: results of the youth mood project. Biol Psychiatry 2009;66:62-68.

Maksimovic J, Phipson B, Oshlack A: A cross-package Bioconductor workflow for analysing methylation array data. F1000Res 2016;5:1281.

Marabita F, Almgren M, Lindholm ME, et al.: An evaluation of analysis pipelines for DNA methylation profiling using the Illumina HumanMethylation450 BeadChip platform. Epigenetics 2013;8:333-346.

McGowan PO, Sasaki A, D'Alessio AC, et al.: Epigenetic regulation of the glucocorticoid receptor in human brain associates with childhood abuse. Nat Neurosci 2009;12:342348.

Murgatroyd C, Patchev AV, Wu Y, et al.: Dynamic DNA methylation programs persistent adverse effects of early-life stress. Nat Neurosci 2009;12:1559-1566.

Non AL, Hollister BM, Humphreys KL, et al.: DNA methylation at stress-related genes is associated with exposure to early life institutionalization. Am J Phys Anthropol 2016;161:84-93.

O'Donovan A, Sun B, Cole S, et al.: Transcriptional control of monocyte gene expression in post-traumatic stress disorder. Dis Markers 2011;30:123-132. 
PsyArXiv post-print doi: 10.31234/osf.io/6ed7n This is the accepted manuscript now published in Psychoneuroendocrinology

Page CM, Vos L, Rounge TB, et al.: Assessing genome-wide significance for the detection of differentially methylated regions. Stat Appl Genet Mol Biol 2018;17.

Pidsley R, CC YW, Volta M, et al.: A data-driven approach to preprocessing Illumina 450K methylation array data. BMC genomics 2013;14:293.

Powell ND, Sloan EK, Bailey MT, et al.: Social stress up-regulates inflammatory gene expression in the leukocyte transcriptome via beta-adrenergic induction of myelopoiesis. Proc Natl Acad Sci U S A 2013;110:16574-16579.

Power C, Thomas C, Li L, et al.: Childhood psychosocial adversity and adult cortisol patterns. The British journal of psychiatry : the journal of mental science 2012;201:199206.

Pruessner JC, Kirschbaum C, Meinlschmid G, et al.: Two formulas for computation of the area under the curve represent measures of total hormone concentration versus timedependent change. Psychoneuroendocrinology 2003;28:916-931.

Reinius LE, Acevedo N, Joerink M, et al.: Differential DNA Methylation in Purified Human Blood Cells: Implications for Cell Lineage and Studies on Disease Susceptibility. Plos One 2012;7.

Ritchie ME, Phipson B, Wu D, et al.: limma powers differential expression analyses for RNAsequencing and microarray studies. Nucleic Acids Res 2015;43:e47.

Rodewald F: Deutsche Bearbeitung des Childhood Trauma Questionnaire: Testbeschreibung und Auswertung. Unveröffentlichtes Manuskript, Medizinische Hochschule Hannover., 2005.

Schwaiger M, Grinberg M, Moser D, et al.: Altered Stress-Induced Regulation of Genes in Monocytes in Adults with a History of Childhood Adversity. Neuropsychopharmacology 2016;41:2530-2540.

Schwaiger M, Heinrichs M, Kumsta R: Oxytocin administration and emotion recognition abilities in adults with a history of childhood adversity. Psychoneuroendocrinology 2018;99:66-71.

Solomon O, Yousefi P, Huen K, et al.: Prenatal phthalate exposure and altered patterns of DNA methylation in cord blood. Environ Mol Mutagen 2017;58:398-410.

Sonuga-Barke E, Kennedy M, Kumsta R, et al.: Child-to-adult neurodevelopmental and mental health trajectories after early life deprivation: the young adult follow-up of the longitudinal English and Romanian Adoptees study. Lancet 2017;389:1539-1548.

Su Y, Cui L, Piao C, et al.: The effects of hematopoietic growth factors on neurite outgrowth. PLoS One 2013;8:e75562.

Suri D, Bhattacharya A, Vaidya VA: Early stress evokes temporally distinct consequences on the hippocampal transcriptome, anxiety and cognitive behaviour. Int $\mathrm{J}$ Neuropsychopharmacol 2014;17:289-301.

Team RC: R: A language and environment for statistical computing; in: R Foundation for Statistical Computing. Vienna, Austria, 2014, vol.

Tingley D, Yamamoto T, Hirose K, et al.: mediation: R Package for Causal Mediation Analysis. J Stat Softw 2014;59.

Turecki G, Meaney MJ: Effects of the Social Environment and Stress on Glucocorticoid Receptor Gene Methylation: A Systematic Review. Biol Psychiatry 2016;79:87-96.

Varricchio L, Tirelli V, Masselli E, et al.: The expression of the glucocorticoid receptor in human erythroblasts is uniquely regulated by KIT ligand: implications for stress erythropoiesis. Stem Cells Dev 2012;21:2852-2865.

Weaver IC, Cervoni N, Champagne FA, et al.: Epigenetic programming by maternal behavior. Nat Neurosci 2004;7:847-854.

Wittchen H-U, Wunderlich U, Gruschwitz S, et al.: Strukturiertes Klinisches Interview für DSM-IV (SKID). Göttingen, Beltz, 1996.

Zhao LR, Navalitloha Y, Singhal S, et al.: Hematopoietic growth factors pass through the blood-brain barrier in intact rats. Exp Neurol 2007;204:569-573. 


\section{Tables and figures}

Table 1. Top ten strongest associations between DNA methylation and childhood trauma $(A)$, and adult cortisol reactivity (B).

A

\begin{tabular}{|c|c|c|c|c|c|c|c|c|c|}
\hline CpG & Gene/ location & B & P-Value & FDR & CpG & Gene/ location & B & P-Value & FDR \\
\hline ch.7.2782052F & EXOC4 & -2.607 & 0.000398 & 0.999 & $\operatorname{cg} 23284931$ & SPON1 & 3.897 & $8.783 \times 10^{-6}$ & 0.996 \\
\hline cg19689211 & KIAA1244 & -2.964 & 0.000578 & 0.999 & $\operatorname{cg} 04788372$ & PFKP & 3.642 & $9.906 \times 10^{-6}$ & 0.996 \\
\hline cg19987219 & $\mathrm{KNCN}$ & -3.474 & 0.000989 & 0.999 & cg08295608 & C14orf79 & 3.6 & $1.626 \times 10^{-5}$ & 0.996 \\
\hline $\operatorname{cg} 10842126$ & PLA2G12B & -3.619 & 0.001152 & 0.999 & $\operatorname{cg} 16453673$ & APPL2 & 3.494 & $1.922 \times 10^{-5}$ & 0.996 \\
\hline cg22877366 & Chromosome 1 & -3.655 & 0.001196 & 0.999 & $\operatorname{cg} 16086416$ & Chromosome 5 & 3.325 & $2.152 \times 10^{-5}$ & 0.996 \\
\hline cg24348107 & OSBPL9 & -3.675 & 0.001222 & 0.999 & cg03293732 & IKZF3 & 3.262 & $2.876 \times 10^{-5}$ & 0.996 \\
\hline cg04458023 & LRP1B & -3.691 & 0.001242 & 0.999 & cg21312412 & VSNL1 & 3.193 & $3.519 \times 10^{-5}$ & 0.996 \\
\hline $\operatorname{cg} 07903677$ & KCNA3 & -3.742 & 0.001311 & 0.999 & $\operatorname{cg} 18181034$ & Chromosome 7 & 2.743 & $4.470 \times 10^{-5}$ & 0.996 \\
\hline cg20622089 & CNR1 & -3.768 & 0.001348 & 0.999 & $\operatorname{cg} 04747382$ & SMPD3 & 2.68 & $6.305 \times 10^{-5}$ & 0.996 \\
\hline cg07368661 & CASKIN2 & -3.874 & 0.001508 & 0.999 & $\operatorname{cg} 02531516$ & Chromosome 1 & 2.629 & $6.656 \times 10^{-5}$ & 0.996 \\
\hline
\end{tabular}


Table 2. Sample characteristics in comparison to previous studies

\begin{tabular}{|c|c|c|c|c|}
\hline Characteristics & Discovery ${ }^{a}$ & Replication $1^{\text {b }}$ & Replication $2^{c}$ & Present Study \\
\hline$N$ & 85 & 45 & 255 & 58 \\
\hline Sex (\% of female) & 50.5 & 80 & 45 & 65.5 \\
\hline Mean Age (range) & 33 (18 to 69$)$ & 28 (19 to 45$)$ & 17 (15 to 18$)$ & 52 (39 to 60) \\
\hline $\begin{array}{l}\text { European descent } \\
(\%)\end{array}$ & 100 & 38 & 100 & 100 \\
\hline Mean CTQ (range) & 31.9 (24 to 63$)$ & 56.8 (25 to 110$)$ & not indicated & 51.46 (25 to 106$)$ \\
\hline $\begin{array}{l}\text { Mean cortisol } \\
\text { AUCi (range) }\end{array}$ & $\begin{array}{c}242.3 \\
(-1030 \text { to } 1876)\end{array}$ & $\begin{array}{c}1185 \\
\text { (378 to 2045) }\end{array}$ & $\begin{array}{c}-37 \\
(-426 \text { to } 313)\end{array}$ & $\begin{array}{c}1489 \\
(-13151 \text { to } \\
18985)\end{array}$ \\
\hline Cortisol assessment & Saliva & Serum & Saliva & Serum \\
\hline $\begin{array}{l}\text { Mean cg27512205 } \\
\text { methylation in \% } \\
\text { (range) }\end{array}$ & $\begin{array}{c}0.15 \\
(0.12 \text { to } 0.19)\end{array}$ & $\begin{array}{c}0.14 \\
(0.11 \text { to } 0.18)\end{array}$ & $\begin{array}{c}0.09 \\
(0.07 \text { to } 0.12)\end{array}$ & $\begin{array}{c}0.12 \\
(0.10 \text { to } 0.14)\end{array}$ \\
\hline $\begin{array}{l}\text { Studied } \\
\text { Tissue (DNA) }\end{array}$ & Whole blood & Whole blood & Buccal cells & $\begin{array}{c}\text { CD14+ } \\
\text { Monocytes }\end{array}$ \\
\hline
\end{tabular}
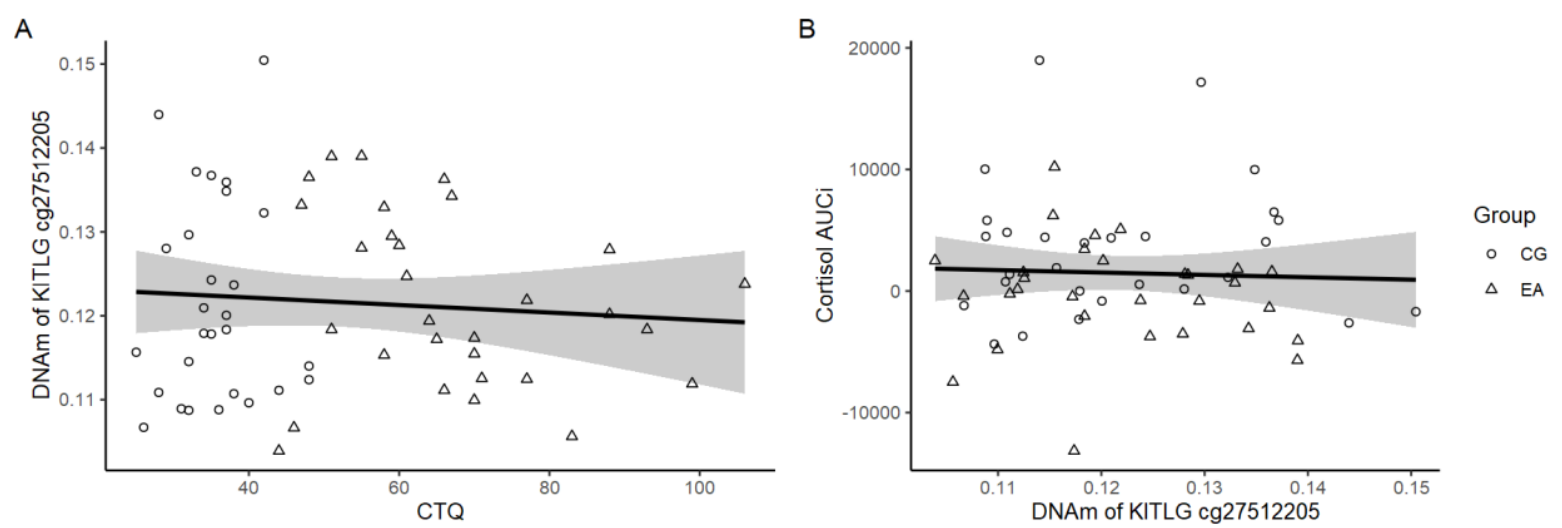

Figure 1. Panel A shows the association between DNA methylation of cg27512205 and CTQ scores. Panel $B$ shows the association between DNA methylation of cg27512205 and cortisol AUCi (both $\beta$ > -0.07 , both $p>0.65)$. EA: early adversity, CG: control group. 


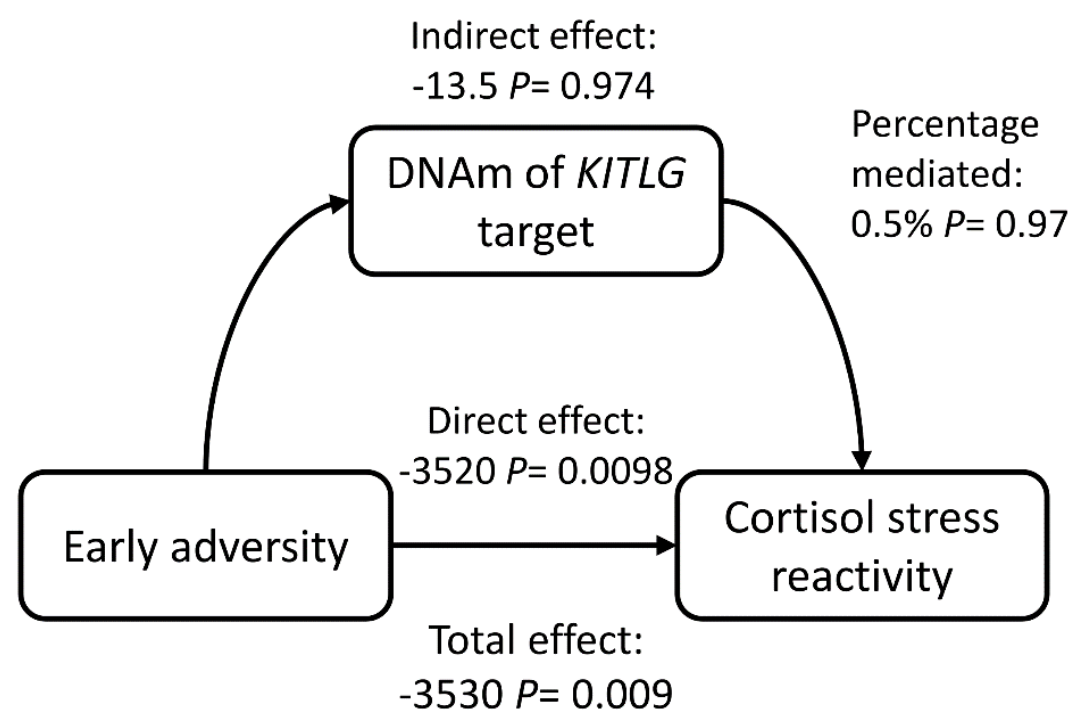

Figure 2. Graphical presentation of the mediation analysis testing the associations between early adversity, DNA methylation of the KITLG locus, and cortisol stress reactivity expressed as AUCi.

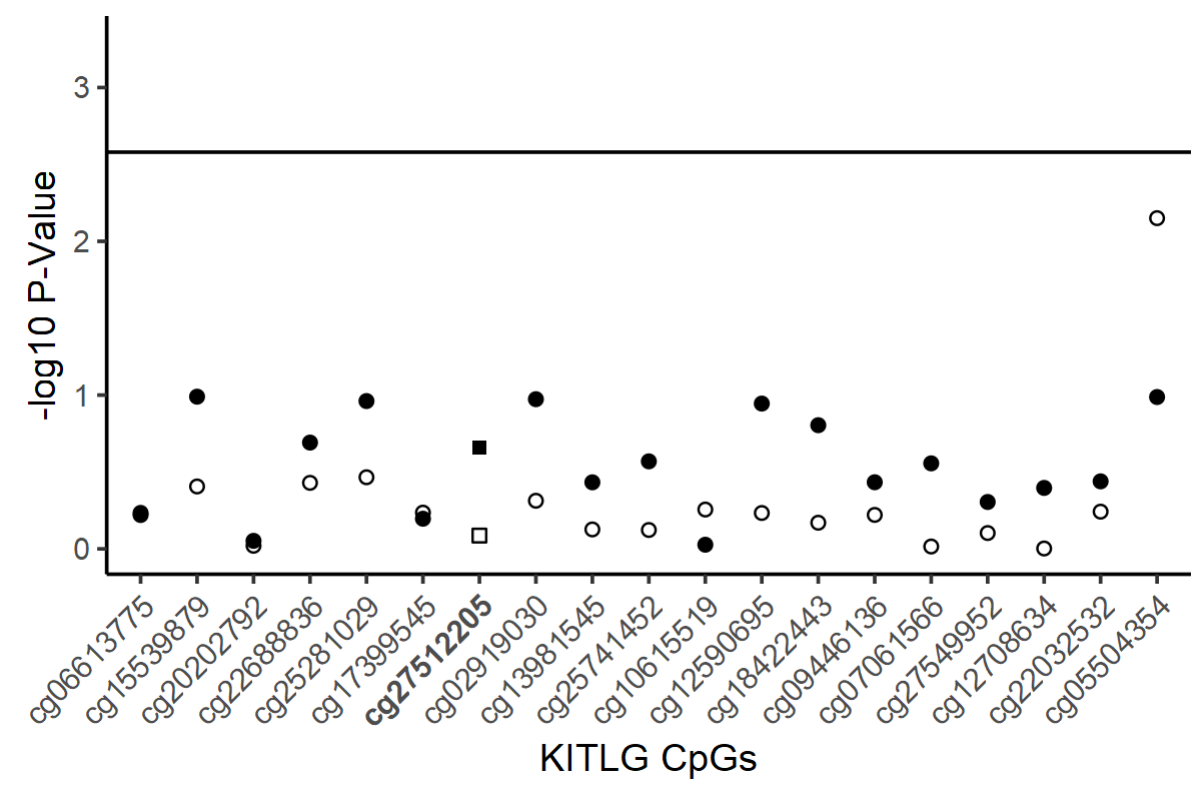

Figure 3. - $\log 10 \mathrm{p}$-values for the association between $19 \mathrm{CpGs}$ annotated to the KITLG locus, CTQ scores (filled circles), and cortisol reactivity (white circles). Squares indicate the target $\mathrm{CpG}$ cg27512205. Solid line indicates the gene-wide corrected level of significance $(p=0.00263)$ 

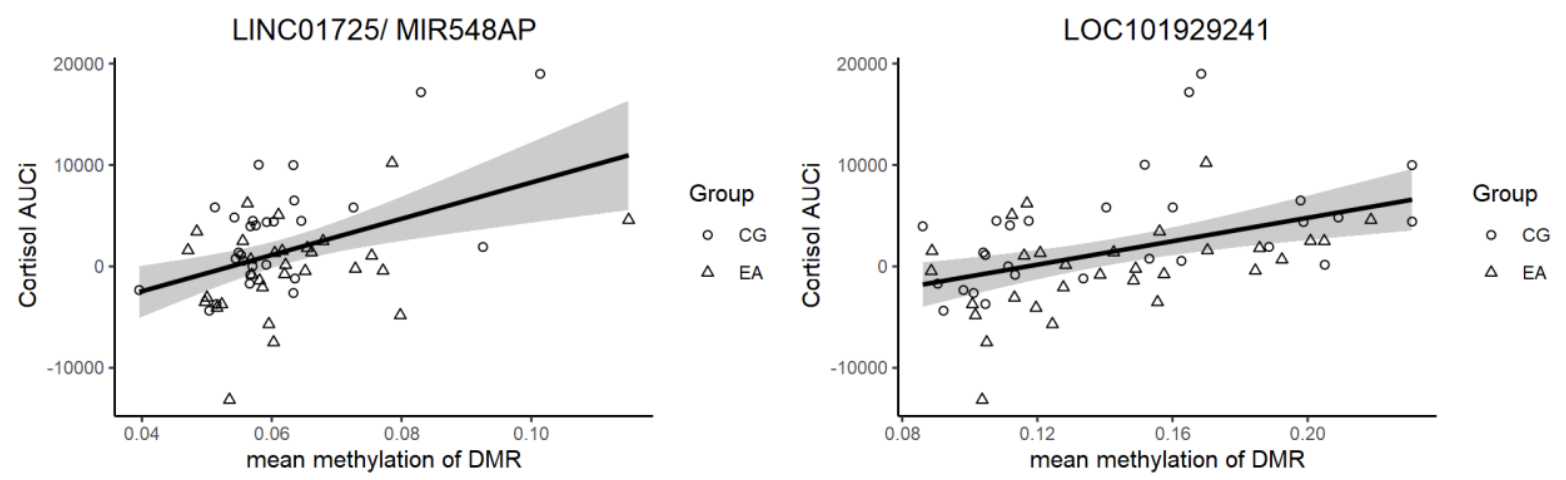

Figure 4. Associations between cortisol stress reactivity and DNA methylation of differentially methylated regions (DMRs). As the CpGs within the DMRs showed strong correlations $(r=.80 \& r=$ .91 , respectively), we calculated the mean DNA methylation values of the DMRs.

\section{Supplementary Material}

Table S1. Frequencies of the subtypes of experienced trauma

\begin{tabular}{|c|c|c|}
\hline & Early adversity & Control group \\
\hline$N$ & 30 & 28 \\
\hline Sex (\% of female) & 67 & 64 \\
\hline Mean CTQ (range) & $67(44$ to 106$)$ & 35 (25 to 48$)$ \\
\hline \multicolumn{3}{|l|}{ CTQ subtypes, n (\%) } \\
\hline - Sexual abuse & $15(50.0)$ & - \\
\hline - Physical abuse & $15(50.0)$ & - \\
\hline - Emotional abuse & $22(73.3)$ & - \\
\hline - Emotional neglect & $26(86.7)$ & - \\
\hline - Physical neglect & $21(70.0)$ & - \\
\hline - Sexual or physical abuse & $23(76.7)$ & - \\
\hline
\end{tabular}




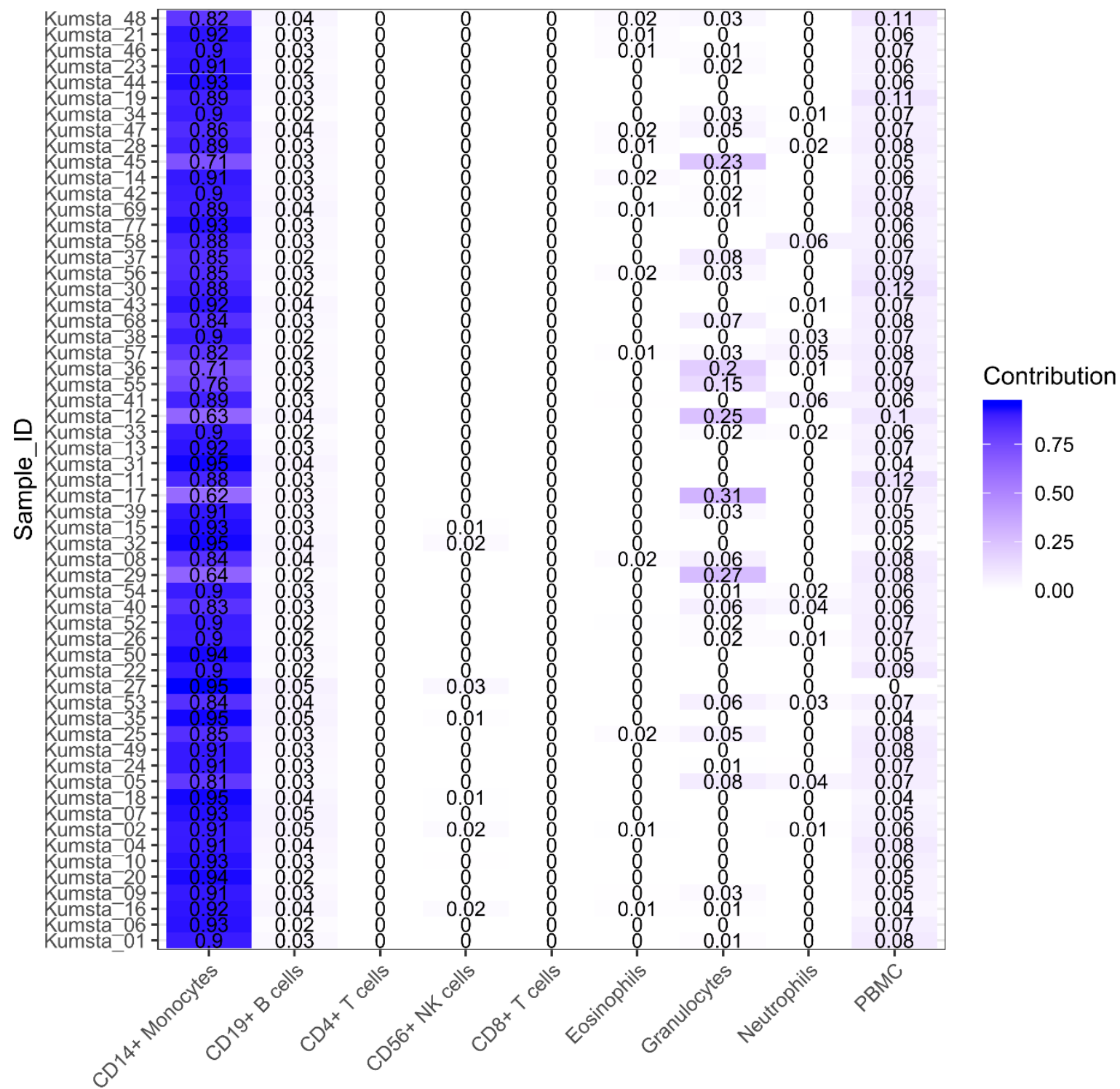

Cell Type

Figure S1. Cell-type composition of the 60 samples. 


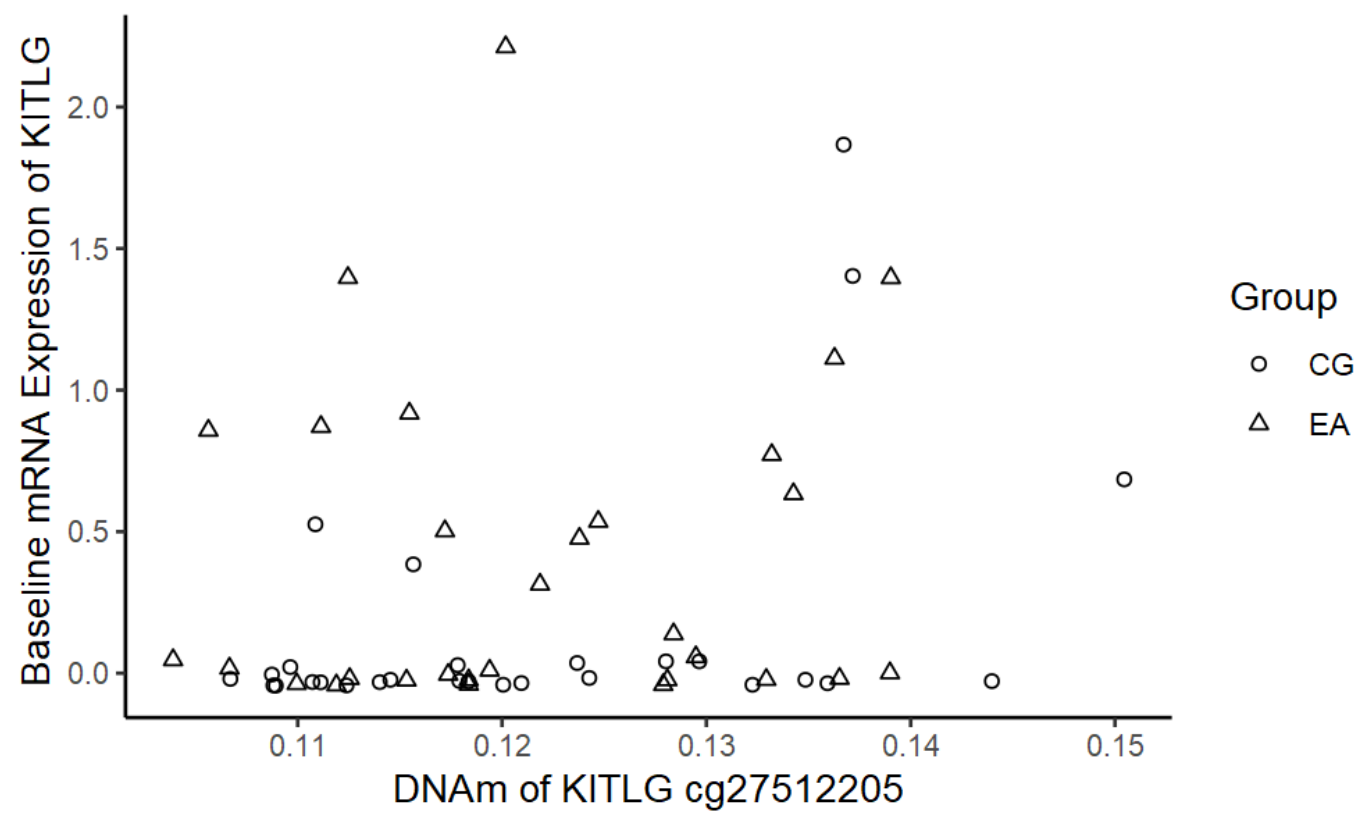

Figure S2. Association between DNA methylation of the target $\mathrm{CpG}$ and baseline KITLG mRNA expression.

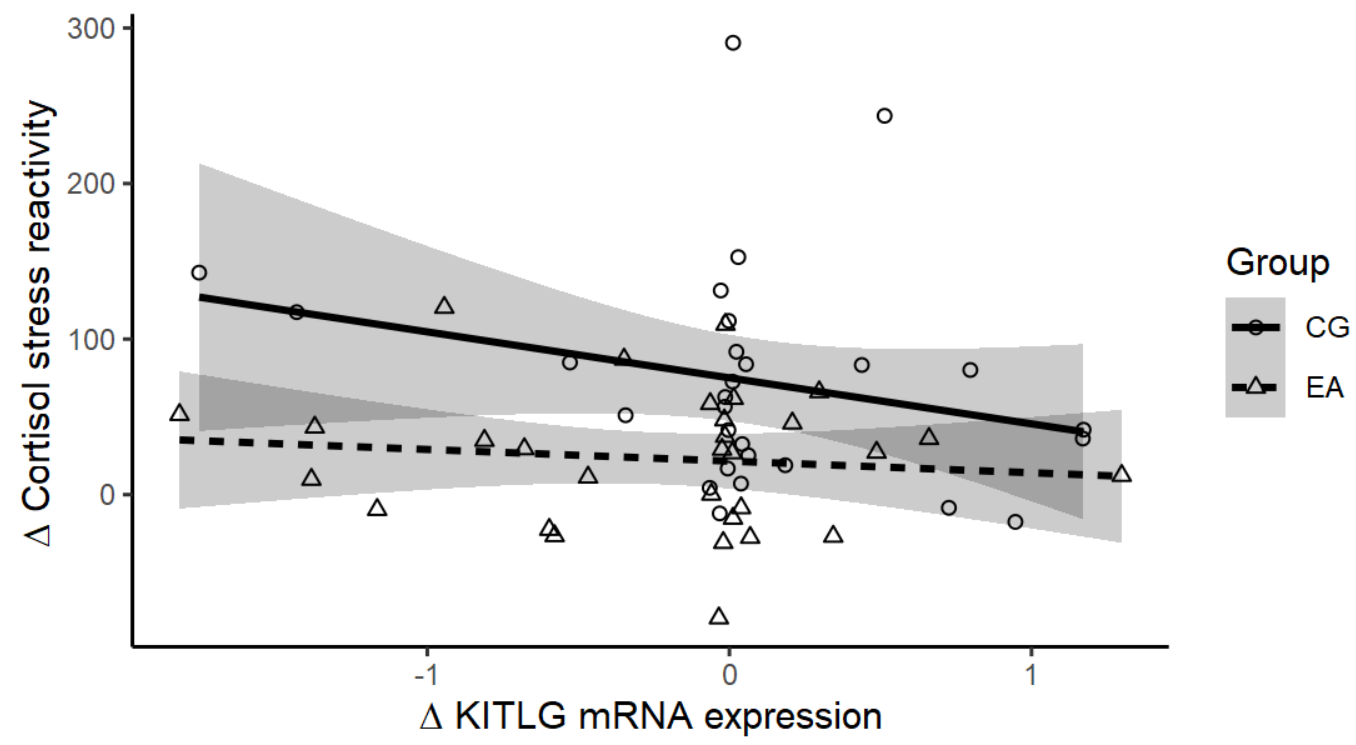

Figure S3. Associations between change in KITLG mRNA expression (180 post stressor minus baseline) and change in cortisol stress reactivity (peak minus baseline). 